\title{
A REGULAR PERTURBATION APPROACH TO SURFACE TENSION DRIVEN FLOWS
}

\author{
Ignacio Da-Riva and Emilio Alvarez Pereira \\ Aerodynamics Laboratory, Polytechnic University of Madrid, Ciudad Universitaria Madrid-3, Spain
}

(Received 3 December 1981)

\begin{abstract}
Surface tension induced convection in a liquid bridge held between two parallel, coaxial, solid disks is considered. The surface tension gradient is produced by a small temperattre gradient parallel to the undisturbed surface.

The study is performed by using a mathematical regular perturbation approach based on a small parameter, $\epsilon$, which measures the deviation of the imposed temperature field from its mean value.

The first order velocity field is given by a Stokes-type problem (viscous terms are dominant) with relatively simple boundary conditions. The first order temperature field is that imposed from the end disks on a liquid bridge immersed in a non-conductive fluid. Radiative effects are supposed to be negligible.

The second order temperature field, which accounts for convective effects, is split into three components, one due to the bulk motion, and the other two to the distortion of the free surface. The relative importance of these components in terms of the heat transfer to or from the end disks is assessed.
\end{abstract}

\section{INTRODUCTION}

Considerable attention has been paid in the last years to surface tension driven convection in liquid bridges, drops and similar configurations, under microgravity conditions. The reason for this interest is that, once reduced buoyancy induced convection by the reduction of the gravity level, the most conspicuous yet outstanding convection driving mechanism will be surface tractions.

The surface tension gradients can be produced either by temperature gradients (thermal convection) or by concentration gradients (solutal convection). This work concerns thermal convection.

The imposed temperature gradient can have a component parallel to and/or a component normal to the undisturbed surface.

The parallel component produces the surface tractions which induce the motion in the bulk through the viscous forces. The motion immediately results whenever a temperature gradient exists, no matter how small.

The normal component of the temperature gradient transfers thermal energy to or from the surface, this would imply a work of the surface tension forces and, thence, motion. But the fluid remains in a state of unstable equilibrium until a critical temperature gradient (better, a critical Marangoni number) is exceeded. This critical value decreases to zero when the surface crisps [1] indicating that in practice both convection driving mechanisms become coupled.

In this paper the imposed temperature gradient is parallel to the undisturbed surface.

Recent contributions to the study of surface tension driven convection can be classified into three categories:

(1) Experimental studies, either using short duration drop-tower techniques [2], or simulating microgravity by the so-called short bridge technique, where the characteristic length in the direction of gravity action is kept as small as feasible [3-8].

(2) Numerical simulation. The complete equations and boundary conditions are solved by computer in order to study the influence of the several relevant parameters [9. 11]. The results are, however, objectionable mainly in connection with the fulfillment of the boundary conditions at the free surface whose position is previously unknown.

(3) Analytical studies of very simple geometrical configurations [12-14]. This approach has been and still is widely used, recall, for example, the Couette-flow approach to boundary layer problems. These types of solution provide a great insight at not excessive cost, and should be pursued before attempting a more sophisticated analysis when the status of knowledge is yet far from satisfactory.

The present approach is midway between 2 and 3 above. The undisturbed geometrical configuration is a cylindrical liquid bridge, of circular cross section, held between two parallel coaxial solid disks. Heat is transferred through these disks.

The most important among the simplifying assumptions introduced in this paper is that the temperature deviates only slightly from its mean value, thence the so-called Marangoni velocity is small, the Reynolds number based on this velocity is also small, and so on. This assumption cannot be applied to real floating zones in melt, but it would be useful in the analysis of many of the foreseen experiments under microgravity with which the authors are familiar.

This paper is arranged as follows:

First the general equations have been written in dimensionless form. These equations are those corresponding to a newtonian viscous liquid, in cylindrical coordinates, in an inertial reference frame. Changes in $\rho$ and $p$ with $T$ are accounted for through the Boussinesq approximation. Boundary conditions at the free surface are discussed to some extent.

The first order approximation for the velocity field is obtained by use of a technique which, although is not 
new, has been recently studied by Joseph [15]. The first order deviation of the free surface from its initial cylindrical shape is then calculated. The deviation becomes infinitely large for the length to diameter ratio corresponding to the Rayleigh stability limit in a cylindrical liquid bridge at rest.

The first order approximation for the temperature field corresponds to the imposed field.

The second order temperature field, which accounts for the first order convective effects is calculated. It is shown that the purely conductive field is modified by (1) the bulk convection induced by surface tension gradients, (2) the effect of surface crispation, and (3) the usually minor influence of surface energy.

The overall heat transfer rate at the end disks can be calculated and compared to that due to conduction alone. It is shown that, in the particular case under consideration (no heat transfer to the environment), the third of the mentioned effects is the sole responsible for the heat transfer enhancement. The first effect cancels, at each disk, because of the symmetry of the axial velocity respect to the mid cross section of the bridge, whereas the second cancels because of the antisymmetry of the free surface shape.

Although, as a general rule, surface tension induced convection should be avoided in space manufacturing processes, it could be augmented for stirring, heat transfer enhancement or observational purposes. This augmentation can be achieved, for a given working liquid, through increased temperature gradients. Nevertheless, only geometrical procedures for enhancing convection are discussed in the present paper.

\section{GOVERNING EQUATIONS IN DIMENSIONLESS FORM}

The equations in cylindrical coordinates, inertial reference frame, for a Newtonian, perfect fluid will be introduced in the following. The geometry, coordinate system and fluid velocity components are shown in Fig. 1.

The variables which appear in the equations below have been made dimensionless with the following characteristic magnitudes.

Length: $L / 2$, Velocity: $U_{r}=-\frac{\left(\frac{\mathrm{d} \sigma}{\mathrm{d} T}\right)_{r} T_{r}}{\mu_{r}}$,

Density: $\rho_{r}$, Temperature: $T_{r}$ Pressure: $\rho_{r} U_{r}^{2}$,

where the subscript $r$ indicates reference values.

The dimensionless parameters which will appear are defined as:

$$
\begin{array}{ll}
\text { Reynolds number: } & R e=\frac{\rho_{r} U_{r} L}{2 \mu_{r}} . \\
\text { Prandtl number: } & \operatorname{Pr}=\frac{c_{r} \mu_{r}}{k_{r}} . \\
\text { Crispation number: } & C r=\frac{\mu_{r} U_{r}}{\sigma_{r} R} .
\end{array}
$$

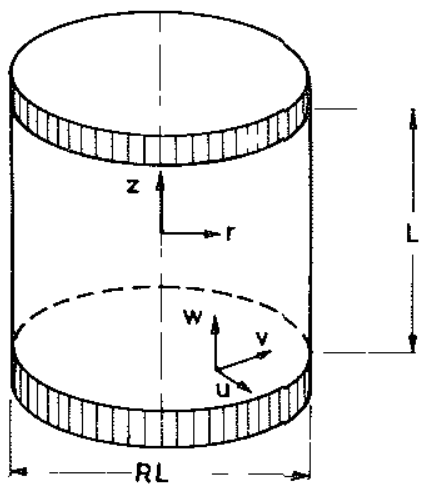

Fig. 1. The liquid bridge with the main nomenclature.

Surface enthalpy to heat conduction ratio, $N_{I}$

$$
N_{I}=-\frac{\left(\frac{\mathrm{d} \sigma}{\mathrm{d} T}\right)_{r} U_{r}}{k_{r}}
$$

It is assumed that thermodynamic and transport properties are temperature independent, viz. $c / c_{r}=k / k_{r}=$ $\mu / \mu_{\mathrm{r}}=\cdots=1$, and that surface tension, $\sigma$, is a linear function of temperature,

$$
\frac{\sigma}{\sigma_{r}}=1-R C r(T-1)
$$

Since temperature is assumed to deviate only slightly from its reference value, $T=1+\epsilon T_{1}+\ldots$, the Boussinesq approximation yields, [16]:

(1) For the density vs temperature variation,

$$
\rho_{1}=-\beta T_{r} T_{1},
$$

where $\beta$ is the thermal expansion coefficient of the fluid. Recall that deviations from the reference values, $\epsilon \rho_{1}$ and $\epsilon T_{1}$, are both dimensionless.

(2) For the pressure gradient term in the momentum equation,

$$
\frac{\nabla p}{\rho}=\frac{\nabla\left(p_{r}+\epsilon p_{1}\right)}{1+\epsilon \rho_{1}}=\left(1+\epsilon \beta T_{r} T_{1}\right) \nabla p_{r}+\epsilon \nabla p_{3}+O\left(\epsilon^{2}\right),
$$

where $p_{r}$ is the pressure, not necessarily uniform, under isothermal conditions. In this particular case, the motion is exclusively induced by temperature gradients in a zero gravity field, thence $\nabla p_{r}=0$.

(3) The continuity equation, which can be written to first order as,

$$
\epsilon \frac{D \rho_{1}}{D t}+\left(1+\epsilon \rho_{1}\right) \nabla \cdot \mathbf{v}+0\left(\epsilon^{2}\right)=0,
$$

becomes that for the incompressible flow.

Finally, it is assumed that Mach number effects are negligible, $\left(\epsilon U_{r}\right)^{2} \ll c_{r} T_{r}$, and that fluid motion is axisymmetrical and stationary, $\partial / \partial \theta=\partial / \partial t=0$. 
With all these simplifying assumptions in mind, the conservation equations become,

Mass

$$
\frac{\partial r u}{\partial r}+\frac{\partial r w}{\partial z}=0
$$

Momentum

$$
u \frac{\partial u}{\partial r}-\frac{v^{2}}{r}+w \frac{\partial u}{\partial z}=-\frac{\partial p}{\partial r}+\operatorname{Re}^{-1}\left\{\frac{\partial^{2} u}{\partial r^{2}}+\frac{1}{r} \frac{\partial u}{\partial r}+\frac{\partial^{2} u}{\partial z^{2}}-\frac{u}{r^{2}}\right\}
$$

$$
\begin{aligned}
u \frac{\partial v}{\partial r}+\frac{u v}{r}+w \frac{\partial v}{\partial z} & =\operatorname{Re}^{-1}\left\{\frac{\partial^{2} v}{\partial r^{2}}+\frac{1}{r} \frac{\partial v}{\partial r}+\frac{\partial^{2} v}{\partial z^{2}}-\frac{v}{r^{2}}\right\} \\
u \frac{\partial w}{\partial r}+w \frac{\partial w}{\partial z} & =-\frac{\partial p}{\partial z}+\operatorname{Re}^{-1}\left\{\frac{\partial^{2} w}{\partial r^{2}}+\frac{1}{r} \frac{\partial w}{\partial r}+\frac{\partial^{2} w}{\partial z^{2}}\right\}
\end{aligned}
$$

Energy

$$
u \frac{\partial T}{\partial r}+w \frac{\partial T}{\partial z}=\operatorname{Re}^{-1} \operatorname{Pr}^{-1}\left\{\frac{\partial^{2} T}{\partial r^{2}}+\frac{1}{r} \frac{\partial T}{\partial r}+\frac{\partial^{2} T}{\partial z^{2}}\right\} .
$$

\subsection{The boundary conditions at the free surface}

Let $T$ be the stress tensor and $\mathbf{n}$ the unit normal to the free surface, assumed to be positive when pointing towards the outer fluid (whose viscosity and thermal conductivity have been neglected).

The normal equilibrium condition at the free surface reads,

$$
\mathbf{n} \cdot \mathrm{T} \cdot \mathbf{n}=-\sigma\left(\frac{1}{R_{1}}+\frac{1}{R_{2}}\right)
$$

where $T \cdot \mathbf{n}$ is the force (viscous and pressure) acting on an element of surface, and $\mathbf{n} \cdot T \cdot \mathbf{n}$ the component of this force normal to the surface. $R_{1}$ and $R_{2}$ are the two principal radii of curvature of the free surface at the point under consideration. The right hand side of the above equation is often called the capillary pressure.

The component of the stress tensor tangential to the free surface is $T \cdot \mathbf{n}-(\mathbf{n} \cdot T \cdot \mathbf{n}) \mathbf{n}$. This vector must balance the surface tractions due to surface tension gradients. Thus,

$$
\mathbf{T} \cdot \mathbf{n}-(\mathbf{n} \cdot T \cdot \mathbf{n}) \mathbf{n}=\nabla \sigma-(\mathbf{n} \cdot \nabla \sigma) \mathbf{n}
$$

Other forces, such as those associated with surface irreversibility, have been neglected.

In addition, a kinematic condition will result from the fact that there is no macroscopic flux of mass through the free surface, whose equation is given by $F(\mathbf{x}, t)=0$

$$
\frac{\partial F}{\partial t}+\mathrm{V} \cdot \nabla F=0
$$

The thermal boundary condition is deduced from the surface balance equation for the internal energy. That equation, which is the surface counterpart of the internal energy equation for the bulk phase, is, [17]:

$$
\frac{\partial \tilde{e}}{\partial t}+\nabla_{t} \cdot\left[\mathbf{V}_{t} \bar{e}\right]=\sigma \nabla_{t} \cdot \mathbf{V}_{t}+\delta\left[\mathbf{n} \cdot \mathbf{J}_{n}\right]
$$

where $\tilde{e}$ is the internal energy per unit surface area. The subscript $t$ refers to a vector projected on the surface, in particular $\nabla_{t}=\nabla-\mathbf{n}(\mathbf{n} \cdot \nabla) . \delta$ is the jump across the surface. $n \cdot \mathbf{J}_{n}$ represents the diffusive flux of total energy to (or from) the surface. Assuming that there is no diffusion of species, $\mathbf{J}_{n}=-k \nabla T$. Effects associated with heat radiation, surface irreversibility, . . . are neglected.

When $\sigma$ depends only on $T$ and is a linear function of $T$, the above equation yields,

$$
T\left(\frac{\mathrm{d} \sigma}{\mathrm{d} T}\right)_{r} \nabla_{t} \cdot \mathrm{V}_{t}=\delta[k \mathbf{n} \cdot \nabla T]
$$

The boundary conditions at the free surface, whose equation in dimensionless form is $r=R[1+l(z)]$, can be written in cylindrical coordinates as:

Dynamic boundary conditions

Normal equilibrium

$$
\begin{aligned}
& p-2 R e^{-1} \frac{1}{1+R^{2}\left(\frac{\mathrm{d} l}{\mathrm{~d} z}\right)^{2}}\left\{\frac{\partial u}{\partial \mathrm{r}}+R^{2}\left(\frac{\mathrm{d} l}{\mathrm{~d} z}\right)^{2} \frac{\partial w}{\partial z}\right. \\
& \left.-R \frac{\mathrm{d} l}{\mathrm{~d} z}\left(\frac{\partial w}{\partial r}+\frac{\partial u}{\partial z}\right)\right\} \\
& =R e^{-1} C r^{-1} \frac{\sigma}{\sigma_{r}}\left\{\frac{1}{R^{2}(1+l)\left(1+R^{2}\left(\frac{\mathrm{d} l}{\mathrm{~d} z}\right)^{2}\right)^{1 / 2}}\right. \\
& \left.-\frac{\frac{\mathrm{d}^{2} l}{\mathrm{~d} z^{2}}}{\left(1+R^{2}\left(\frac{\mathrm{d} l}{\mathrm{~d} z}\right)^{2}\right)^{3 / 2}}\right\}
\end{aligned}
$$

Axial equilibrium

$$
\begin{gathered}
\frac{1}{\left(1+R^{2}\left(\frac{\mathrm{d} l}{\mathrm{~d} z}\right)^{2}\right)^{1 / 2}}\left\{2 R \frac{\mathrm{d} l}{\mathrm{~d} z}\left(\frac{\partial u}{\partial r}-\frac{\partial w}{\partial z}\right)\right. \\
\left.+\left(1-R^{2}\left(\frac{\mathrm{d} l}{\mathrm{~d} z}\right)^{2}\right)\left(\frac{\partial w}{\partial r}+\frac{\partial u}{\partial z}\right)\right\}=-\left\{\frac{\partial T}{\partial z}+R \frac{\mathrm{d} l}{\mathrm{~d} z} \frac{\partial T}{\partial r}\right\} .
\end{gathered}
$$

Azimuthal equilibrium

$$
r \frac{\partial}{\partial r}\left(\frac{v}{r}\right)-R \frac{\mathrm{d} l}{\mathrm{~d} z} \frac{\partial v}{\partial z}=0
$$

Kinematic boundary condition

$$
u=w R \frac{\mathrm{d} l}{\mathrm{~d} z} .
$$


Thermal boundary condition

$$
\begin{aligned}
\frac{\partial T}{\partial r}- & R \frac{\mathrm{d} l}{\mathrm{~d} z} \frac{\partial T}{\partial z}=-N_{I} \frac{T}{\left(1+R^{2}\left(\frac{\mathrm{d} l}{\mathrm{~d} z}\right)^{2}\right)^{1 / 2}}\left\{\frac{\partial w}{\partial z}\right. \\
& \left.+R \frac{\mathrm{d} l}{\mathrm{~d} z}\left(\frac{\partial u}{\partial z}+\frac{\partial w}{\partial r}\right)+R^{2}\left(\frac{\mathrm{d} l}{\mathrm{~d} z}\right)^{2} \frac{\partial u}{\partial r}+R \frac{\mathrm{d}^{2} l}{\mathrm{~d} z^{2}} u\right\}
\end{aligned}
$$

The boundary conditions at the end disks, $z= \pm 1$, are the following:

$$
u=v=w=0,
$$

and either $T$ or $\partial T / \partial z$ prescribed. In this particular case we will impose the value

$$
T(r, \pm 1)=1 \pm \epsilon T_{w}
$$

\section{THE FIRST ORDER APPROXIMATION}

Since the imposed dimensionless temperature field deviates only slightly from the reference value, 1 , it is inferred that, (1) the dimensionless velocities are of order $\epsilon$ (in particular, $v=0$ because of the axisymetry of the configuration), and (2) the free surface deviates only slightly from its undisturbed cylindrical shape.

Introducing $u=\epsilon u_{\mathrm{f}}, v=0, w=\epsilon w_{1}$, and $l=\epsilon l_{1}$ in the above set of equations and boundary conditions, and neglecting higher order terms, the following Stokes-type problem results.

Mass

$$
\frac{\partial r u_{1}}{\partial r}+\frac{\partial r w_{1}}{\partial z}=0
$$

Momentum

$$
\begin{aligned}
& \operatorname{Re} \frac{\partial p_{1}}{\partial r}=\frac{\partial^{2} u_{1}}{\partial r^{2}}+\frac{1}{r} \frac{\partial u_{1}}{\partial r}+\frac{\partial^{2} u_{1}}{\partial z^{2}}-\frac{u_{1}}{r^{2}} \\
& \operatorname{Re} \frac{\partial p_{1}}{\partial z}=\frac{\partial^{2} w_{1}}{\partial r^{2}}+\frac{1}{r} \frac{\partial w_{1}}{\partial r}+\frac{\partial^{2} w_{1}}{\partial z^{2}} .
\end{aligned}
$$

Energy

$$
\frac{\partial^{2} T_{1}}{\partial r^{2}}+\frac{1}{r} \frac{\partial T_{1}}{\partial r}+\frac{\partial^{2} T_{1}}{\partial z^{2}}=0
$$

with the following boundary conditions at the free surface, which can be transferred to $r=R$ within the first order approximation.

Normal equilibrium

$$
p_{r}=\frac{1}{R e C r R^{2}}=\frac{2 \sigma / R L}{\rho_{r}}
$$

and

$$
\frac{\mathrm{d}^{2} l_{1}}{\mathrm{~d} z^{2}}+\frac{l_{\mathrm{I}}}{R^{2}}=C r\left[2 \frac{\partial u_{1}}{\partial r_{r=R}}-\operatorname{Re} p_{1}-\frac{T_{1}}{R}\right]
$$

The first of these two equations merely indicates that the reference pressure is that corresponding to static equilibrium. The second equation will be used to calculate the axial deviation of the free surface from its undisturbed position.

Axial equilibrium

$$
\frac{\partial w_{1}}{\partial r}+\frac{\partial u_{1}}{\partial z}=-\frac{\partial T_{1}}{\partial z}
$$

which illustrates how the surface tension gradients induce the motion in the bulk through the viscous forces.

Kinematic boundary condition

$$
u_{1}=0
$$

Thermal boundary condition

$$
\frac{\partial T_{1}}{\partial r}=-N_{I} \frac{\partial w_{1}}{\partial z} .
$$

Here it is seen that the free surface is stretched as a consequence of the thermal energy transferred to it.

The boundary conditions at the end disks are the same as above.

The resulting problem is linear. The temperature field becomes decoupled in the sense that the heat transfer is purely conductive and can be deduced without resorting to the velocity field. Thus, $T=1+\epsilon T_{w} z$.

\subsection{The first order velocity field}

Introducing the stream function for axisymmetrical flows, $\chi$, defined as

$$
u_{1}=\frac{\partial \chi}{\partial z} ; \quad w_{1}=-\frac{\partial \chi}{\partial r}-\frac{\chi}{r}
$$

the problem for $\chi$ reduces to the biharmonic differential equation

$$
\left(\frac{\partial^{2}}{\partial r^{2}}+\frac{1}{r} \frac{\partial}{\partial r}+\frac{\partial^{2}}{\partial z^{2}}-\frac{1}{r^{2}}\right)^{2} \chi=0
$$

with the boundary conditions

$$
\begin{gathered}
r=R, \quad \frac{\partial^{2} \chi}{\partial r^{2}}+\frac{1}{r} \frac{\partial \chi}{\partial r}-\frac{1}{r^{2}} \chi=T_{w} ; \quad \frac{\partial \chi}{\partial z}=0 . \\
z= \pm 1, \quad \chi=\frac{\partial \chi}{\partial z}=0 .
\end{gathered}
$$

There is at present an elegant mathematical tool allowing the solution of biharmonic problems in series form. It was first used in Elasticity by Smith (1952) (quoted in [15]), by Lee and Fung[18] in Stokes-type problems and, fairly recently by Joseph[15], Joseph and Sturges [19], who are particularly concerned with the convergence of the series. This tool will be used to obtain a formal 
solution of our problem even though the convergence of the resulting series are not yet clearly established.

3.1.1 Biharmonic eigenfunctions. These are the socalled Papkovich-Fadle functions. The even functions are defined[15] as:

$$
\left(\begin{array}{c}
\phi_{1}^{(n)}(z) \\
\phi_{2}^{(n)}(z)
\end{array}\right)=\left(\begin{array}{c}
S_{n} \sin S_{n} \cos S_{n} z-S_{n} z \cos S_{n} \sin S_{n} z \\
-\phi_{1}^{(n)}(z)-2 \cos S_{n} \cos S_{n} z
\end{array}\right),
$$

odd functions are also given in [15] but are irrelevant here.

The functions $I_{1}\left(S_{n} r\right) \phi_{1}^{(n)}(z)$, where $I_{1}$ is the modified Bessel function of order one, are biharmonic, i.e. solutions of (7).

The boundary conditions at the end disks are satisfied, since $\phi_{1}^{(n)}( \pm 1)=0$, provided that

$$
\frac{\mathrm{d} \phi_{1}^{(n)}(z)}{\mathrm{d} z_{z= \pm 1}}=0
$$

and this furnishes the eigenvalues $S_{n}$, which are the first-quadrant complex roots of $\sin 2 S+2 S=0$.

In our particular case, the imposed temperature field is odd in $z, u=\partial \chi / \partial z$ must be odd and, thence, $\chi$ even.

3.1.2 The stream function $\chi \cdot \chi(r, z)$ will be expressed as

$$
\chi(r, z)=\sum_{-\infty}^{\infty} \frac{C_{n}}{S_{n}^{2}} I_{1}\left(S_{n} r\right) \phi_{1}^{(n)}(z)
$$

The boundary conditions ( 8 ) become respectively

$$
\begin{aligned}
& \frac{\partial^{2} \chi}{\partial r^{2}}+\frac{1}{r} \frac{\partial \chi}{\partial r}-\frac{\chi}{r^{2}}=\sum_{-\infty}^{\infty} C_{n} I_{1}\left(S_{n} R\right) \Phi_{1}^{(n)}(z)=T_{w} \\
& \frac{\partial \chi}{\partial z}=0 \rightarrow \frac{\partial^{2} \chi}{\partial z^{2}}=\sum_{-\infty}^{\infty} C_{n} I_{1}\left(S_{n} R\right) \Phi_{2}^{(n)}(z)=0 .
\end{aligned}
$$

According to [15] the coefficients, $C_{n}$, in a series representation

$$
\left(\begin{array}{l}
f(z) \\
g(z)
\end{array}\right)=\sum_{-\infty}^{\infty} C_{n}\left(\begin{array}{l}
\Phi_{1}^{(n)}(z) \\
\Phi_{2}^{(n)}(z)
\end{array}\right)
$$

are given by

$$
\begin{aligned}
C_{n}=-\frac{1}{4 \cos ^{4} S_{n}} \int_{-1}^{1}\left\{\left(\Phi_{1}^{(n)}(z)+2 \cos S_{n}\right.\right. & \left.\cos S_{n} z\right) g(z) \\
& \left.+\Phi_{1}^{(n)}(z) f(z)\right\} \mathrm{d} z
\end{aligned}
$$

The expression for the stream function then becomes:

$$
\chi(r, z)=-T_{w} \sum_{-\infty}^{\infty} \frac{1}{\cos ^{4} S_{n}} \frac{1}{S_{n}^{2}} \frac{I_{1}\left(S_{n} r\right)}{I_{1}\left(S_{n} R\right)} \Phi_{1}^{(n)}(z)
$$

Figure 2 shows the first order streamlines, $r \chi=$ Const., for three different values of the slenderness, $1 / R$, of the liquid bridge.

3.1.3 The velocity components and pressure field. The velocity components, as deduced from (9), are:

$$
\begin{aligned}
& u_{1}=\frac{\partial \chi}{\partial z}=-T_{w} \sum_{-\infty}^{\infty} \frac{1}{\cos ^{2} S_{n}} \frac{1}{S_{n}^{2}} \frac{I_{1}\left(S_{n} r\right)}{I_{1}\left(S_{n} R\right)} \frac{\mathrm{d} \Phi_{1}^{(n)}(z)}{\mathrm{d} z} \\
& w_{1}=-\frac{\partial \chi}{\partial r}-\frac{\chi}{r}=T_{w} \sum_{-\infty}^{\infty} \frac{1}{\cos ^{4} S_{n}} \frac{1}{S_{n}} \frac{I_{0}\left(S_{n} r\right)}{I_{1}\left(S_{n} R\right)} \Phi_{1}^{(n)}(z) .
\end{aligned}
$$

In particular, the axial compunent $w_{1}(R, z)$ at the free surface is shown in Fig. 3 for several values of $R$. Notice that $w_{1}$ decreases when the slenderness, $1 / R$, of the liquid bridge increases.

The pressure field can be deduced from the couple of momentum equations (2a) in the usual way. The final result is:

$$
R e p_{1}(r, z)=-2 T_{w} \sum_{-\infty}^{\infty} \frac{1}{\cos ^{3} S_{n}} \frac{I_{0}\left(S_{n} r\right)}{I_{1}\left(S_{n} R\right)} \sin S_{n} z
$$

\subsection{The shape of the free surface}

This shape is deduced from the differential equation (4a) with the attachment condition at the disk edge $\left(l_{3}( \pm 1)=0\right)$. The condition of liquid volume invariance is automatically satisfied, to first order, since $l_{1}(z)$ is odd in $z$. The final expression is too complicated to be written down here, but several results have been plotted in Fig. 4.

It can be shown that $l_{1}(z)$ becomes infinitely large when $\sin 1 / R=0$. The smallest root of this equation is precisely the Rayleigh stability limit for a sylindrical liquid bridge at rest.

\section{THE SECOND ORDER TEMPERATURE FELD}

The next term in the expression of $T, T=$ $1+\epsilon T_{w} z+\epsilon^{2} T_{2}(r, z)$, is calculated from the energy equation (3), boundary condition (6), and $T_{2}(r, \pm 1)=0$ at the end disks. Retaining terms up to order $\epsilon^{2}$, the following differential equation for $T_{2}$ results:

$$
\frac{\partial^{2} T_{2}}{\partial r^{2}}+\frac{1}{r} \frac{\partial T_{2}}{\partial r}+\frac{\partial^{2} T_{2}}{\partial z^{2}}=\operatorname{RePr} T_{w} w_{1}
$$

In order to write down the boundary condition at $r=R$, $N_{I}$ has been expressed as $N_{I}=\epsilon^{i} N(j \geq 1, N \simeq 0(1))$ accounting for the fact that $N_{I}$ is usually small [20]. Then (6) becomes,

$$
\frac{\partial T_{2}}{\partial r_{r=R}}=R T_{w} \frac{\mathrm{d} l_{1}}{\mathrm{~d} z}-\epsilon^{i-1} N \frac{\mathrm{d} w_{1}}{\mathrm{~d} z},
$$

where the last terms disappears unless $j=1$.

The above linear problem will be split into three, giving respectively the effects of bulk convection $\left(T_{2 b}\right)$, surface crispation $\left(T_{2 c}\right)$, and surface energy $\left(T_{2 n}\right)$.

\subsection{The effect of bulk convection}

The first problem will be defined by the differential equation (3b) with no heat transfer through the cylindrical surface $r=R$ and zero temperature at the end disks. Since $T_{2 b}$ is even in $z$, the overall heat transfer through either disk must be zero. 


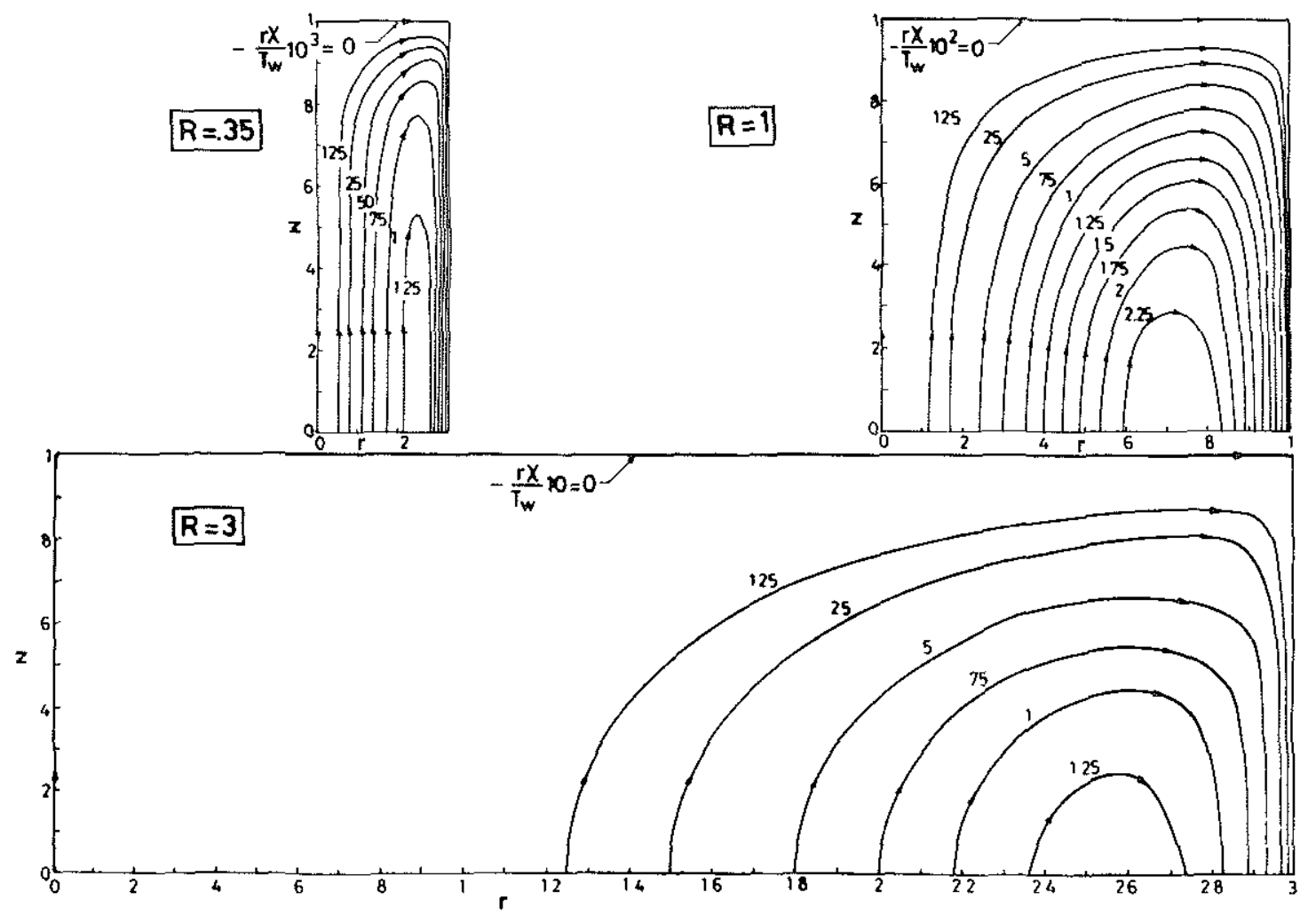

Fig. 2. Streamlines corresponding to the first order velocity field for three different values of the slenderness, $1 / R$, of the liquid bridge.

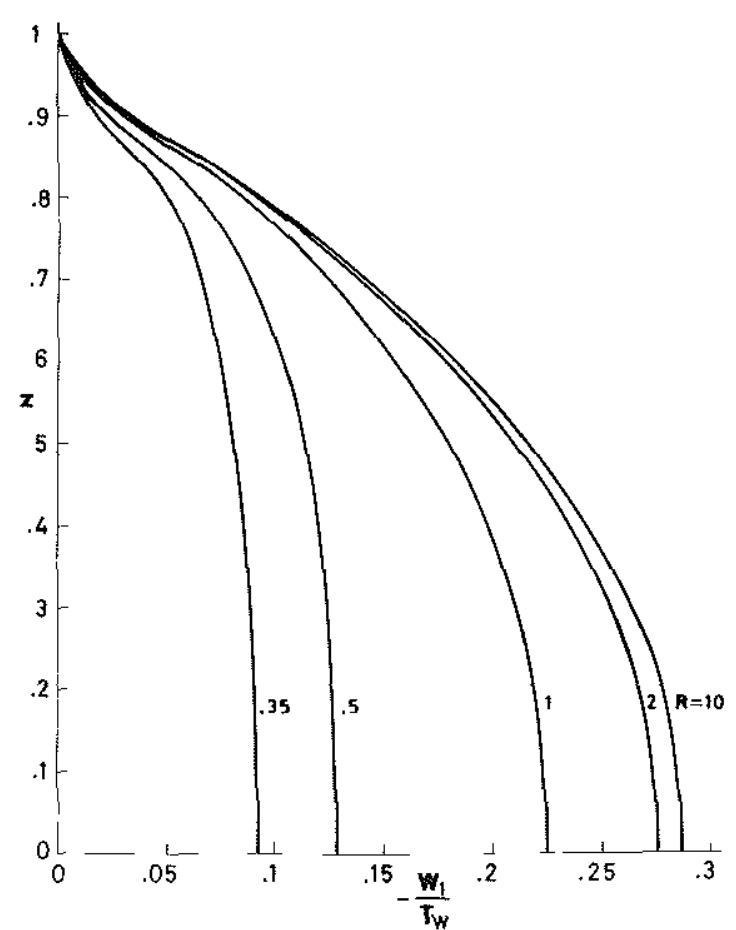

Fig. 3. The first order axial velocity at the free surface, $w_{1}(R, z)$, for several values of the slenderness, $1 / R$, of the liquid bridge.

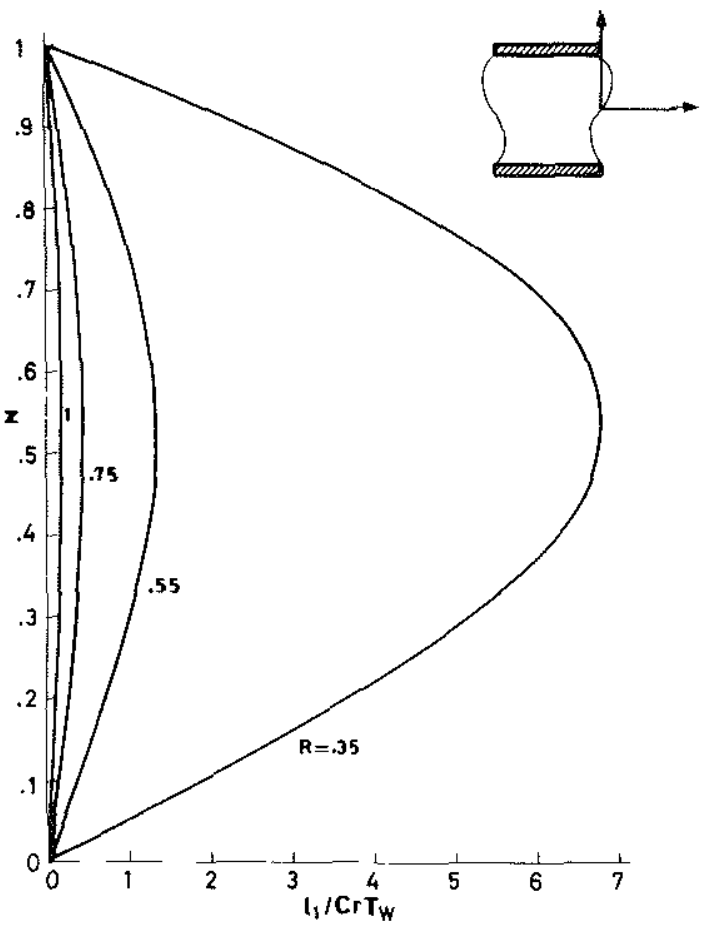

Fig. 4. The radial deviation of the free surface from its origfnal cylindrical configuration, $l_{1}(z)$, for several values of the slenderness, $1 / R$, of the liquid bridge. 
A particular solution of (3b), vanishing at $z= \pm 1$ is:

$$
\begin{aligned}
T_{2 p}= & \frac{\operatorname{RePr} T_{w}^{2}}{4} \sum_{-\infty}^{\infty} \frac{1}{\cos ^{4} S_{n}} \frac{1}{S_{n}^{3}} \frac{I_{0}\left(S_{n} r\right)}{I_{1}\left(S_{n} R\right)}[(1 \\
& \left.\left.+2 \sin ^{2} S_{n}\right) \Phi_{\mathrm{t}}^{(n)}(z)-\frac{S_{n}}{2}\left(z^{2}-1\right)\left(\Phi_{1}^{(n)}(z)+\Phi_{2}^{(n)}(z)\right)\right]
\end{aligned}
$$

It can be seen[21] that at $r=R$,

$$
\frac{\partial T_{2 p}}{\partial r_{r=R}}=\frac{\operatorname{RePr} T_{w}^{2}}{8}\left(1-z^{2}\right) .
$$

Now, if the temperature field, $T_{2 b}$, due to the effect of bulk convection is split into

$$
T_{2 b}=T_{2 p}+T_{2 h}
$$

the problem for $T_{2 h}$ will be:

$$
\frac{\partial^{2} T_{2 h}}{\partial r^{2}}+\frac{1}{r} \frac{\partial T_{2 h}}{\partial r}+\frac{\partial^{2} T_{2 h}}{\partial z^{2}}=0
$$

with

$$
\begin{gathered}
r=R \quad \frac{\partial T_{2 h}}{\partial r_{r=R}}=-\frac{\operatorname{Re} \operatorname{Pr} T_{w}^{2}}{8}\left(1-z^{2}\right), \\
z= \pm 1 \quad T_{2 h}(r, \pm 1)=0 .
\end{gathered}
$$

The solution of this problem is straightforward [22],

$$
\begin{gathered}
T_{2 h}=-\frac{8}{\pi^{4}} \operatorname{RePr} T_{w}{ }^{2} \sum_{n=0}^{\infty} \frac{(-1)^{n}}{(2 n+1)^{4}} \\
\quad \times \frac{I_{0}\left(\frac{2 n+1}{2} \pi r\right)}{I_{1}\left(\frac{2 n+1}{2} \pi R\right)} \cos \frac{2 n+1}{2} \pi z .
\end{gathered}
$$

The convergence of this expression is extremely fast and a single term will suffice to provide an accurate result.

Figure 5 shows $T_{2 b}=T_{2 p}+T_{2 h}$, as deduced from (12) and (16), in the form of temperature profiles for several values of the coordinate $z$.

\subsection{The effect of surface crispation}

The problem for the temperature field due to surface crispation, $T_{2 c}$, can be solved as that for $T_{2 h}$ above. Unfortunately we were, up to now, unable to express in closed form the function $l_{1}(z)$ and some sort of numerical approximation should be used. The expression

$$
l_{1}(z)=l_{\max } \sin \pi z
$$

provides an excellent approximation [21] except for short zones which, on the other hand, remain practically cylindrical.

With the above approximation for $l_{3}(z), T_{2 c}$ becomes

$$
\begin{gathered}
T_{2 c}=-\frac{8}{\pi} R T_{w} l_{\max } \sum_{0}^{\infty} \frac{(-1)^{n}}{(2 n+1)(2 n+3)} \\
\quad \times \frac{I_{0}\left(\frac{2 n+1}{2} \pi r\right)}{I_{0}\left(\frac{2 n+1}{2} \pi R\right)} \cos \frac{2 n+1}{2} \pi z .
\end{gathered}
$$

The overall heat transfer rate through the end disks is again zero. Since there is no heat flux through the mid cross-section,

$$
\int_{0}^{R} 2 \pi r \frac{\partial T_{2 c}}{\partial z_{z= \pm 1}} \mathrm{~d} r=-2 \pi R^{2} T_{w} \int_{0}^{ \pm 1} \frac{\mathrm{d} l_{1}}{\mathrm{~d} z} \mathrm{~d} z=0
$$

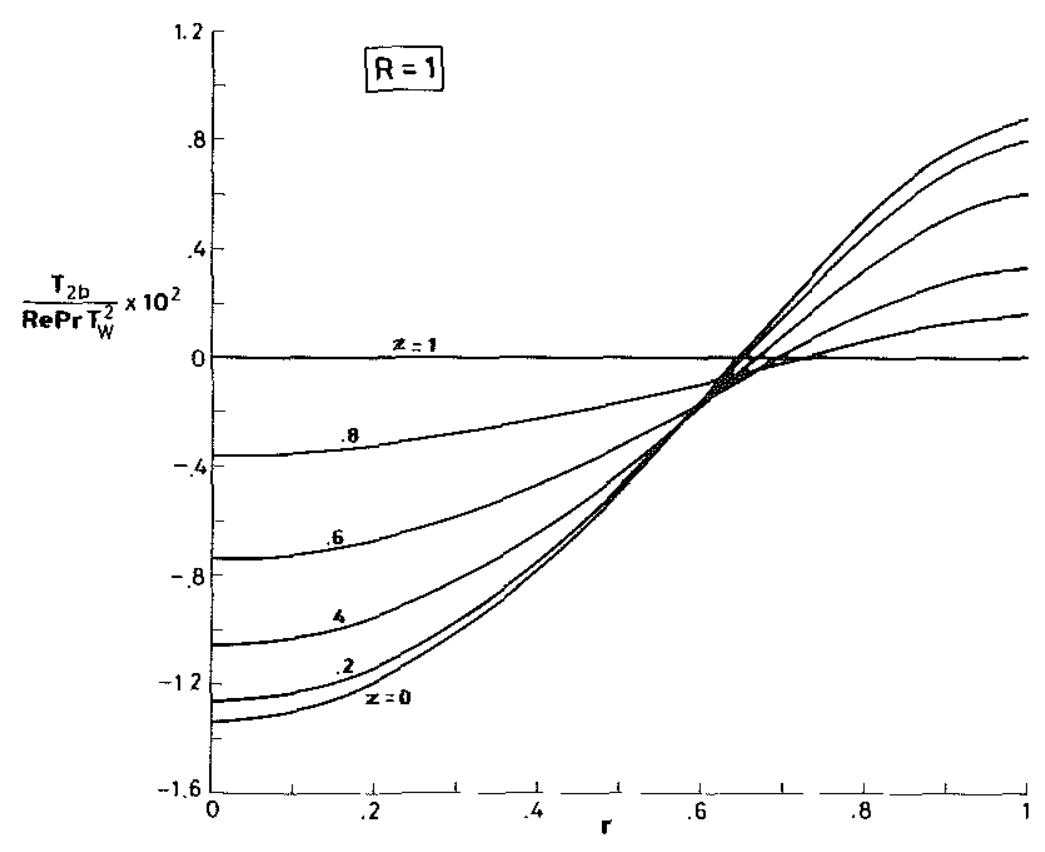

Fig. 5. The effect of bulk convection on the second order temperature field, $T_{2 b}(R=1)$. 


\subsection{The effect of surface energy}

This problem is again defined by the Laplace differential equation with,

$$
\begin{array}{ll}
r=R, & \frac{\partial T_{2 n}}{\partial r_{r=R}}=-N \frac{\partial w}{\partial z}, \\
z= \pm 1, & T_{2 n}(r, \pm 1)=0 .
\end{array}
$$

In this case the temperature field results to be odd in $z$. A formal expression for $T_{2 n}$ is,

$$
T_{2 n}=N \sum_{i}^{\infty}\left[\int_{-1}^{1} w(R, \xi) \cos n \pi \xi \mathrm{d} \xi\right] \frac{l_{0}(n \pi r)}{I_{1}(n \pi R)} \sin n \pi z .
$$

The heat transfer rate through any cross-section, $z$, may be written as

$$
\int_{0}^{R} 2 \pi r \frac{\partial T_{2 n}}{\partial z} \mathrm{~d} r=2 \pi R N\left[w(R, z)-\int_{-1}^{1} w(R, \xi) \mathrm{d} \xi\right] .
$$

In particular, for the end discs,

$$
\int_{0}^{R} 2 \pi r \frac{\partial T_{2 n}}{\partial z_{z= \pm 1}} \mathrm{~d} r=-2 \pi R N \int_{-1}^{1} w(R, \xi) \mathrm{d} \xi .
$$

Thence, this term is the only one which contributes to the overall heat transfer through the end disks, under the validity of the present model.

\section{CONCLUSIONS}

A method to analyze low Reynolds number surface tension driven flows has been presented. It is seen that:

(1) The dimensionless axial velocity at the free surface decreases when the slenderness of the bridge increases, because of the dragging effect of viscosity. When convection is to be enhanced, bridges of annular cross section can be used. For a given outer $R$, annular configurations present larger surfaces, although the axial velocity will decrease to zero with the thickness of the annular liquid wall.

(2) The stability limit for the slenderness of the bridge is that corresponding to static equilibrium.

(3) When the thermal conductivity of the fluid which surrounds the bridge can be neglected, the convective heat transfer through the end disks is very small, and is only due to surface energy effects. Although this conclusion is based on the assumption that the imposed temperature field is odd in $z$ (antisymmetrical), matters do not differ markedly when the imposed field is even. It can be seen, for example, that the contribution of $T_{2 b}$ is nil in any case.
Acknowledgement-We wish to acknowledge the support by the Spanish Space Research Commission (CONIE).

\section{REFERENCES}

1. K. A. Smith, On convective instability induced by surfacetension gradients. J. Fluid Mech. 24, 401-414 (1966).

2. S. Ostrach and A. Pradhan, Surface-tension induced convection at reduced gravity. AIAA $J$. 16, $419-424$ (1978).

3. $\mathrm{Ch} . \mathrm{H}$. Chun, Marangoni convection in a floating zone under reduced gravity. $J$. Crystal Growth 48, 600-610 (1980).

4. Ch. H. Chun and W. Wuest, Suppression of temperature oscillations of thermal Marangoni Convection in a floating zone by superimposing of rotating flows. Acta Astronautica 9, 225-230 (1982).

5. D. Schwabe, A. Scharmann and F. Preisser, Studies of Marangoni convection in crystal growth melts. $X X X I$ Cong. $I A F$, Tokyo, Preprint IAF-80-C-140. Pergamon Press, Oxford (1980).

6. D. Schwabe, F. Preisser and A. Scharmann, Verification of the oscillatory state of thermocapillary convection in a floating zone under low gravity. Acta Astronautica, 9, 265-273 (1982).

7. R. Monti and L. G. Napolitano, Experimental validation of kinematic and thermal scaling laws in Marangoni flows. $X X X I$ Cong. IAF, Tokyo, Preprint IAF-80-C-138. Pergamon Press, Oxford (1980).

8. R. Monti, Experimental equipment for the measurement of thermofluidynamic conditions in fluid physics experiments on board of space platform. Acta Astronautica 9, 275-284 (1982).

9. C. E. Chang and W. R. Wilcox, Analysis of surface tension driven flow in floating zone melting. J. Heat Mass Transfer 19, 355-366 (1976).

10. R. Piva and M. Strani, Numerical solutions for surface tension driven flows. $X X X I$ Cong. IAF, Tokyo, Preprint IAF-80-C-112. Pergamon Press, Oxford (1980).

11. G. Graziani, M. Strani and R. Piva, Effect of free surface radiation in axisymmetric thermocapillary flows. Acta Astronautica 9, 231-243 (1982).

12. L. G. Napolitano and C. Golia, Coupled Marangoni boundary layers. Acta Astronautica 8, 417-434 (1981).

13. L. G. Napolitano and C. Golia, Buoyancy effects in Marangoni boundary layers. XXXII Cong. IAF, Roma, Preprint IAF-81-135, Pergamon Press, Oxford (1981).

14. L. G. Napolitano, Temperature field in Marangoni-Poiseuille plane flows, $X X X I$ Cong. IAF, Tokyo, Preprint IAF-80-C110. Pergamon Press, Oxford (1980).

15. D. D. Joseph, The convergence of biorthogonal series for biharmonic and Stokes flow edge problems. Part I. SIAM J. Appl. Math. 33, 337-347 (1977).

16. L. D. Landau and E. M. Lifshitz, Fluid Mechanics, 1st Edn, pp. 212-215. Pergamon Press, Oxford (1959).

17. L. G. Napolitano, Thermodynamics and dynamics of surface phases. Acta Astronautica 6, 1093-1112 (1979).

18. J. S. Lee and Y. C. Fung, Stokes flow around a circular cylindrical post confined between two parallel plates. $J$. Fluid Mech, 37, 657-670 (1969).

19. D. D. Joseph and L. Sturges, The convergence of biorthogonal series for biharmonic and Stokes flow edge problems. Part II. SIAM J. Appl. Math. 34, 7-26 (1978).

20. R. Monti, Study on Fluid Phenomena Influencing the Design of Zero-g-Experiments, pp. 89-91. Techno System Report TS-11-79, Naples (1979).

21. I. Da-Riva, Microgravity fluid dynamics-some developments. CISM Udine, Advanced School, pp. 8.5-8.13 (1981).

22. H. S. Carslaw and J. C. Jaeger, Conduction of Heat in Solids, 6th Edn, pp. 217-225. Oxford University Press (1967). 